\title{
Referees 2012
}

Through their evaluation of manuscripts, the referees contribute greatly to the scientific standard of our journal. As a sign of our appreciation of this unselfish activity, we have decided to publish a list of referees. The following referees reviewed papers that were published in volume 222:

\begin{tabular}{|c|c|c|}
\hline \multirow{2}{*}{\multicolumn{3}{|c|}{$\begin{array}{l}\text { Aboobaker A } \\
\text { Amemiya S }\end{array}$}} \\
\hline & & \\
\hline Andersen $\mathrm{S}$ & Kaslin J & Qiu GF \\
\hline Angerer LM & Kikuchi Y & \\
\hline Arendt D & Kiomoto M & Rebecchi L \\
\hline \multirow[t]{2}{*}{ Asami T } & Koehler A & Riechmann V \\
\hline & Kramer E & Rushlow C \\
\hline Baker N & Kühl M & \\
\hline Batistoni R & Kuo DH & Scaal M \\
\hline Beebe D & Kurokawa D & Schlosser G \\
\hline Bely A & & Schmidt-Ott U \\
\hline Bosch T & Leung TC & Schneider S \\
\hline Braeunig P & Litt A & Schwager E \\
\hline Hidalgo A & Liu Q & Seaver E \\
\hline Brand-Saberi B & Lynch J & Shi DL \\
\hline Bruce A & & Siomi M \\
\hline Bruneau B & Mallo M & Smith A \\
\hline \multirow[t]{2}{*}{ Bucher G } & Martin A & Sonawane M \\
\hline & Martinez AS & Steinbeisser $\mathrm{H}$ \\
\hline \multirow[t]{2}{*}{ Cai Y } & Minelli A & Stollewerk A \\
\hline & Morgan J & \\
\hline \multirow[t]{2}{*}{ Frank U } & Muller A & Technau U \\
\hline & Murakami R & Thewissen JGM \\
\hline Gould A & & Tomoyasu Y \\
\hline \multirow[t]{2}{*}{ Grillner S } & Nakamura A & \\
\hline & Nakao H & Vasiliauskas D \\
\hline Han $\mathrm{C}$ & Nardi J & \\
\hline Hatta M & Nath U & Wada H \\
\hline Henry J & Newmark P & Wang J \\
\hline \multirow[t]{2}{*}{ Holstein $\mathrm{T}$} & Nicolas FJ & Wang Z \\
\hline & Nieto A & \\
\hline Jacobs D & & Yaguchi S \\
\hline Janssen R & Pechmann M & Yan $\mathrm{H}$ \\
\hline Jiao R & Pera E & \\
\hline Jin $P$ & Philpott A & Zhang Y \\
\hline Jindra M & Plickert G & Zoran M \\
\hline
\end{tabular}

